\title{
A Case Report on Congenital Ichthyosis - Collodion Baby
}

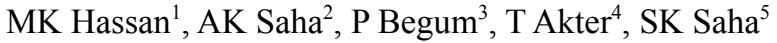

\begin{abstract}
:
Collodion baby describes a highly characteristic clinical entity in newborns encased in a yellowish translucent membrane resembling collodion. In most cases the condition either precedes the development of one of a variety of ichthyoses, the commonest of which are lamellar ichthyosis and non-bullous ichthyosiform erythroderma, or occasionally represents an initial phase of other ichthyoses such as ichthyosis vulgaris. In at least $10 \%$ of all cases of collodion baby, the condition is followed by a mild ichthyosis of lamellar type, so mild as to be considered more or less normal, so-called self-healing collodion baby or 'lamellar ichthyosis of the newborn'. In this report, we present a severe form of ichthyosis.
\end{abstract}

Key words: Ichthyosis, Lamellar, Harlequin ichthyosis, Newborn diseases, Skin diseases.

\section{Introduction:}

The term collodion baby (CB) (lamellar desquamation/ exfoliation of the newborn), describes a highly characteristic clinical entity in newborns encased in a yellowish translucent membrane resembling collodion. In most cases the condition either precedes the development of one of a variety of ichthyosis, the commonest of which are the autosomal recessive, rarely autosomal dominant forms of lamellar ichthyosis and non-bullous ichthyosiform erythroderma, or occasionally represents an initial phase of other ichthyoses such as ichthyosis vulgaris, X-linked ichthyosis, Netherton's syndrome, neutral lipid storage disease or the Sjögren-Larsson syndrome. In at least $10 \%$ of all cases, the collodion baby phase is followed by a mild ichthyosis of lamellar type, so mild as to be considered more or less normal, so-called self healing collodion baby or 'lamellar ichthyosis of the newborn'.

1. Dr. Md. Kamrul Hassan, MBBS, DCH (Paediatrics), Junior Consultant, Department of Paediatrics, Faridpur Medical College Hospital, Faridpur.

2. Dr. Aloke Kumar Saha, MBBS; FCPS (Paediatrics), Associate Professor \& Head, Department of Paediatrics, Faridpur Medical College, Faridpur.

3. Dr. Poly Begum, MBBS; FCPS (Obs \& Gynae), Assistant Professor, Department of Obstetrics \& Gynaecology, Diabetic Association Medical College, Faridpur.

4. Dr. Tahmina Akter, MBBS, MD (Paediatrics), Associate professor, Dept. of Paediatrics, Diabetic Association Medical College, Faridpur.

5. Dr. Shyamol Kumar Saha, MBBS, DCH, Senior Consultant, Dept of Paediatrics, FMCH, Faridpur.

Address of correspondence :

Dr. Md. Kamrul Hassan, MBBS, DCH, Junior Consultant, Dept. of Paediatrics, FMCH, Faridpur. Mob: +8801913389808.

E-mail: hassankamrul007@gmail.com
Although self-healing collodion baby was firstly thought to be an autosomal recessive condition, it is most likely genetically heterogeneous ${ }^{1}$. Since 1884, when Hallopeau used the term collodion baby for the first time, about 270 cases have been sporadically reported including familial, self healing cases and localized forms ${ }^{2-4}$.

\section{Case report:}

We present a child, born as a result of the first, controlled and uneventful, consanguineous pregnancy. A female infant was born at 37 weeks of gestation. The 1- and 5-minute Apgar scores were 9, respectively. The newborn's birth weight was $3570 \mathrm{~g}$ ( $90^{\text {th }}$ centile), birth length $49 \mathrm{~cm}\left(98^{\text {th }}\right.$ centile) and head circumference $35 \mathrm{~cm}\left(91^{\text {st }}\right.$ centile). From the Obstetrics ward the baby was transferred to the Special Care Neonatal Unit (SCANU) of Faridpur Medical College Hospital. On admission, the newborn presented with normal vital signs, generalized edema, anxiety. We evaluates three items - facial expressions, limb movements, and vocal expression. Whole body surface was covered with a yellowish membrane with laminar cracks especially on the neck. The eyelids were everted, edematous with profuse mucus secretion. The earlobes were deformed by swelling and tension of the skin, the lips were everted showing eclabion. Due to the collodion membrane, limited range of motion in all joints was present. The result of physical assessment was otherwise normal. During the first week of life, laboratory findings such as erythrocytic sedimentation rate, C-reactive protein, complete blood count as well 
as other relevant biochemical findings were in normal ranges. Ultrasound findings of the upper abdomen, brain and echocardiography were normal. TORCH (serological analysis for toxoplasmosis, rubella, cytomegalovirus, herpes simplex) IgM was negative. Karyotype showed 46, XX (normal). Since septicemia is a common complication, regular skin, eye, nose, external ear canal and throat swabs, as well as blood, stool and gastric aspirate cultures, were done for bacteria and candida, and they were all negative. Upon admission, parenteral administration of antibiotics (ampicillin, gentamicin) and analgesics (paracetamol, fentanyl) was initiated. The baby was nursed in humidified incubator (60 to $70 \%)$, with close monitoring of body temperature. The skin was treated with emollients every 4 hours during the first day of life. After the first week of life, when almost all the membrane has desquamated (except on the palms and soles), emollients were applied twice a day. A prophylactic, topical antibiotic cream was applied on fissures and bare skin. Artificial eye drops were initiated (ectropion from the $2^{\text {nd }}$ day of life) and periorbital area was treated.

An increase risk of infections both cutaneous and systemic like candida and bacterial, fissures, ischemia and oedema of limbs due to membrane compression. The first line of management is moisturizers and topical keratolytic agents, they enhances skin barrier and facilitate desquamation. Sodium chloride, urea, vitamin E acetate, glycerol and petroleum jelly are various agents available as moisturizers and lubricants. These infants are at increased risk of intoxication by absorption of topical products, like salicylates or keratolytics due to impaired skin. In severe cases with marked hyperkeratosis keratolytical agents like lactic acid, glycolic acid, salicyclic acid, N- acetyl- cystine, glycol can be used. Ectropion is managed by application of artificial tears and eye lubricants. Administration of retinoids have keratolytic effect and help in elimination of scales and prevent hyperkeratosis of skin. Isotretinoin and retinoids both have proven to be effective in these cases. External auditory canal must be regularly cleaned by an ENT person so that accumulation of scales is prevented and so hearing loss can be prevented. Enteral feeding was started on the first day of life, initially via nasogastric tube, later by bottle. Due to high transdermal fluid loss, intravenous fluid, $250 \mathrm{ml} / \mathrm{kg} / \mathrm{day}$, and nasogastric fluid supplementation were commenced. One episode of hypernatremia was registered, probably as a result of technical incident. The written informed consent was taken from parents for case reporting and image publication.

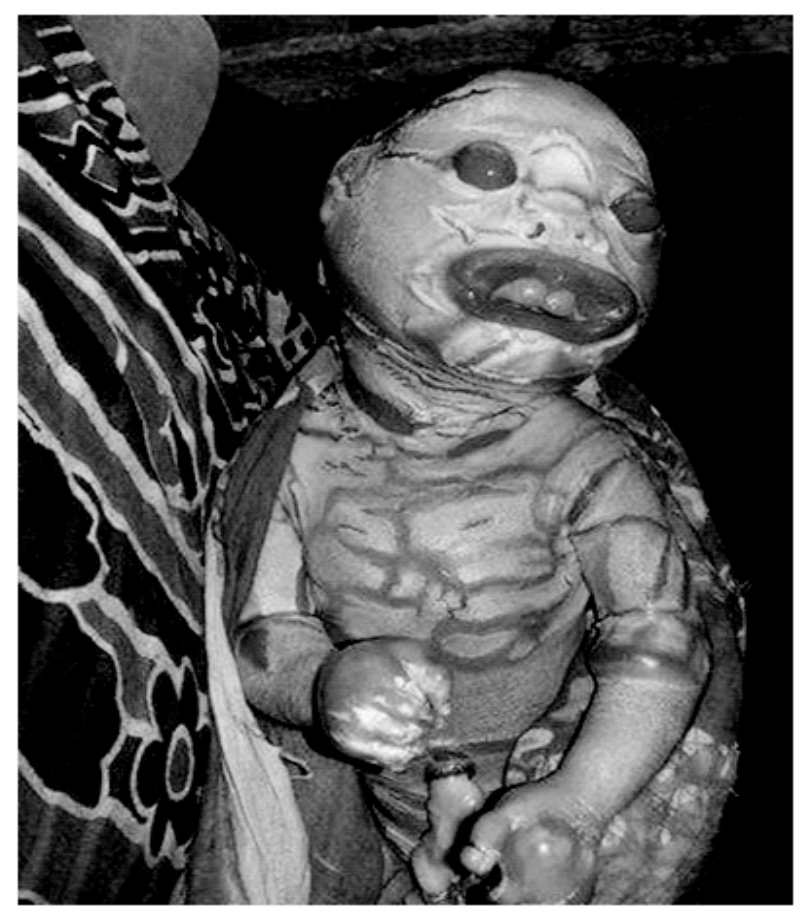

Figure-1: Collodion baby at birth

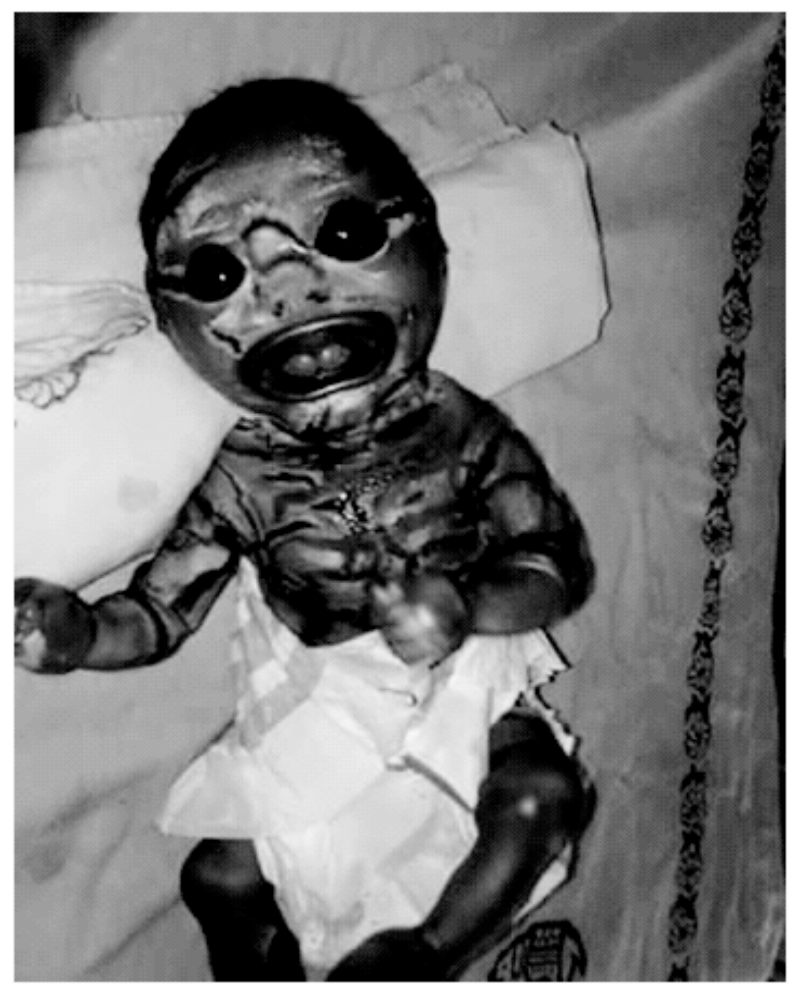

Figure-2: Figure showing generalized erythema and oedema over the body. 


\section{Discussion:}

Collodion membrane is a result of disturbed epidermal lipid and protein homeostasis. It has the same etiopathogenesis as ichthyosiform erythroderma, which develops in the majority of collodion babies. Most of collodion babies have autosomal recessive congenital ichthyosiform erythrodermas as a result of functional mutations in transglutaminase 1 - TGM1, ALOXE3 or ALOX12B genes, ABCA12, HIPAL4/ ichthyin, ABHD5 or other genes responsible for aforementioned epidermal homeostasis ${ }^{5,6}$. Most cases develop ichtyosis, ichtyosis-like conditions or other keratinization disorder. Most cases, approximately $75 \%$ will go on to develop autosomal recessive congenital ichtyosis, either lamellar ichthyosis or congenital ichthyosiform erythroderma - particularly non-bullous. After shedding the collodion membrane, the skin, especially on the trunk, usually remains dry and resembles a mild form of ichthyosis vulgaris. Only $10 \%$ of cases eventually develop normal skin. This is known as self-healing collodion baby ${ }^{7-9}$. Self-healing collodion baby is caused by compound heterozygous mutations which in utero render the transglutaminase 1 inactive cis form; after delivery, a normal phenotype develops, since in the extrauterine environment the enzyme isomerizes back to its active trans form. About $15 \%$ of cases are caused by different conditions which include keratinization disorders, such as ichthyosis vulgaris and trichothiodystrophy-ichthyosis syndrome, SjogrenLarsson syndrome, ichthyosis variegata, palmoplantar keratoderma with anogenital leukokeratosis, as well as non-syndromic autosomal dominant loricrin keratoderma, anhidrotic ectodermal dysplasia etc ${ }^{10}$. Collodion babies are often born prematurely (between 32 and 36 weeks of gestation) and are small for gestational age. However, premature delivery is not usually a significant problem. Our patient was born in term, with normal somatometric parameters. During the first weeks of life, the diagnosis is unmistakable. Collodion baby is a syndrome in which a bright red newborn is encased in a taut, shiny, yellowish translucent membrane resembling collodion, known as collodion membrane. This extra layer of skin usually covers the whole body and looks tight; it may be described as 'plastic skin', 'parchment-like' or 'as if dipped in hot wax'. The whole body surface is covered by armorlike plates. The face appears immobilized. Tension around the eyes and the mouth results in: ectropion and eversion of the lips (eclabion) as in our patient, producing a rather fish-like appearance of the mouth, and effacement of the nose and ears. The nostrils may be blocked. Since the baby's eyes and mouth are forced opened, if appropriate treatment is not started, keratitis due to xerophthalmia and subsequent blindness may occur ${ }^{11}$. Severe tightness of the skin on the thorax may interfere with respiration, and very occasionally, respiratory distress may be caused by nasal obstruction, such as impaired feeding and breathing. Since respiration can be also compromised during delivery, due to aspiration of amniotic fluid with squamous debris, this can be seen on fetal ultrasound and early delivery by caesarean section can prevent this complication. The membrane may cause physical constraints of underlying tissues with development of constriction bands with subsequent reduction of local blood supply and swelling of extremities ${ }^{12,13}$. Awareness of potential complications is of utmost importance. The baby should be nursed in an incubator at high humidity, with constant monitoring of body temperature. High humidity $(40-100 \%)$ is exceptionally important in order to decrease the level of transepidermal fluid loss, that is 6 to 7 folds increased in these newborns ${ }^{14}$. On the other hand, high humidity increases the risk of skin colonization with different pathogens. Other supportive measures include maintenance of fluid and electrolyte balance. In severe cases, intravenous fluid therapy in indicated, but in less severe cases oral or nasogastric fluid supplementation is recommended. Peritoneal dialysis may be indicated if renal failure occurs. The skin should be maintained soft in order to decrease scaling. Other treatment options include pain alleviation (for example paracetamol or opioids), as well as use of emollients and mild topical steroids in order to decrease inflammation, and application of artificial tear drops. Use of emollients is contradictory $^{15}$. The collodion membrane should not be peeled-off roughly. Bands of tight skin constricting digits, hands or feet may occasionally require surgical division. Our patient was initially treated with emollients 6 times a day, and twice a day after the first week of life.

In collodion babies with localized lesions, local use of retinoic acid and calcipotriol is a good treatment option. Also, introduction of systemic retionids in the treatment of generalized lesions was highly successful leading to significant decrease in mortality, from 50\% in the $1960 \mathrm{~s}$ to $11 \%$ in 1986 , and less than $5 \%$ nowadays ${ }^{15}$. As large transepidermal fluid loss results in high metabolic demands, supplementation of calories is mandatory in early phases of treatment in order to maximize the growth potential. Therefore, as a result of sucking difficulties due to eclabium, feeding via nasogastric tube is initially recommended. In most cases, the membrane starts to dry and breaks within the first 48 hours of life and usually peels off completely in two to four weeks like in our case, but may reform several times. Subsequently, the typical features of one of several above mentioned varieties of ichthyosis gradually develop over a period of weeks or months, but it was not the case in our patient in whom, after the collodion membrane peeled off, the skin became smooth, pink and without scales. Although the necessity for intensive care depends on the baby's condition, most are discharged within the first 4 weeks, once the membrane completely peels off. No correlation between the severity of ichthyosis that follows and the initial severity in a collodion baby has been reported. Since on light microscopy 
histopathological findings of skin biopsy within the first weeks are identical, regardless if severe ichthyosis will develop or not, and cannot distinguish which disturbance will develop, the diagnosis of collodion baby is clinical. Apart from orthokeratotic stratum corneum, light microscopy of the skin shows normal epidermis and dermis. Regardless of the clinical status which may still be indistinguishable, it is possible to make a prediction on histological grounds at about 15 days, but the best option is to perform skin biopsy after the collodion membrane is peeled off. Electron microscopy features vary in the early neonatal phase and may persist for several months with no predictive value $^{15}$. Although skin biopsy was not performed in our patient, because the parents have not consented to the intervention, based on visible characteristics of the baby's skin over the follow-up, it seems quite reasonable to consider this case as a rare self-healing collodion baby. Moreover, at present, it seems more likely that apart from relevant investigations including genetic family counseling, only observation over a period of months will clarify the fate of the skin in this newborn.

\section{Conclusion :}

The treatment is mainly supportive like use of incubators and IV fluids and tube feeding and use of emollients. In our experience, we can suggest that reassurance to the parents is needed that the newborn can be survived and condition will get better. Special care to the skin is needed and collodion membrane should not be peeled off, it will shed after 1-2 week and infection should be prevented.

\section{References :}

1. Paige DG, Gennery AR, Cant AJ. The neonate. In: Burns T, Breathnach S, Cox N, Griffi ths C, editors. Rook's Textbook of Dermatology. $8^{\text {th }}$ ed. Oxford: Wiley-Blackwell; 2010. p. 17.117.85 .

2. Van Gysel D, Lijnen RL, Moekti SS, de Laat PC, Oranje AP. Collodion baby: follow-up study of 17 cases. J Eur Acad Dermatol Venerol. 2002; 16:472-5.

3. Judge MR. Collodion baby and harlequin is hthyosis. In: Harper J, Oranje A, Prose N, editors. Texbook of pediatric dermatology. $2^{\text {nd }}$ ed. Malden: Blackwell Publishing; 2006. p. 118-5.

4. Tuzun Y, Iscimen A, Pehlivan O. Collodion baby. J Turk Acad Dermatol. 2008; 2(2):82201.

5. Judge MR, McLean WHI, Munro CS. Disorders of keratinization. In: Burns T, Breathnach S, Cox N, Griffi ths C, editors. Rook`s Textbook of Dermatology. $8^{\text {th }}$ ed. Oxford: Wiley-Blackwell; 2010. p. 19.1-19.122.

6. Frenk E, de Techtermann F. Self-healing collodion baby: evidence for autosomal recessive inheritance. Pediatr Dermatol. 1992; 9:95-7.

7. Raghunath M, Hennies HC, Ahvazi B, Vogel M, Reis A, Steinert PM, et al. Self healing collodion baby: a dynamic phenotype explained by a particular transglutaminase 1 mutation. J Invest Dermatol. 2003; 120:224-8.
8. Carbajal R, Paupe A, Hoenn E, Lenclen R, Olivier Martin M. DAN: une échelle comportementale d'évaluation de la douleur aiguë du nouveau-né. Arch Pediatr 1997; 4(7):623-8.

9. Harting M, Brunetti-Pierri N, Chan CS, Kirby J, Dishop MK, Richard G, et al. Self-healing collodion membrane and mild nonbullous congenital ichthyosiform erythroderma due to 2 novel mutations in the ALOX12B gene. Arch Dermatol. 2008; 144(3):351-6.

10. Langer K, Konrad K, Weninger M, Wolff K. Collodion baby with transition to mild lamellar ichthyosis. Clinical course, histopathology and ultrastructural findings. Hautarzt 1991; 42:34-8.

11. Sybert VP. Disorders of the epidermis. In: Sybert VP, editor. Genetic skin disorders. New York, Oxford: Oxford University Press; 1997. p. 5-128.

12. Roberts JB, Adelson D. Case report: prolonged collodion membrane causing constrictive bands of the digits and treatment. Dermatol Online J. 2010; 16(1):15.

13. Sandler B, Hashimoto K. Collodion baby and lamellar ichthyosis. J Cutan Pathol. 1998; 25(2):116-21.

14. Taieb A, Labreze C. Collodion baby: what's new. J Eur Acad Dermatol Venereol. 2002; 16:436-7.

15. Prado R, Ellis LZ, Gamble R, Funk T, Arbuckle HA, Bruckner AL. Collodion baby: an update with a focus on practical management. J Am Acad Dermatol. 2012; 67:1362-74. 